Subscriber access provided by UNIV OF CAMBRIDGE

\title{
Article
}

\section{Controlling the self-assembly behavior of aqueous chitin nanocrystal suspensions}

Aurimas Narkevicius, Lisa M. Steiner, Richard M. Parker, Yu Ogawa, Bruno Frka-Petesic, and Silvia Vignolini Biomacromolecules, Just Accepted Manuscript • DOI: 10.1021/acs.biomac.9b00589 • Publication Date (Web): 04 Jun 2019

Downloaded from http://pubs.acs.org on June 6, 2019

\section{Just Accepted}

"Just Accepted" manuscripts have been peer-reviewed and accepted for publication. They are posted online prior to technical editing, formatting for publication and author proofing. The American Chemical Society provides "Just Accepted" as a service to the research community to expedite the dissemination of scientific material as soon as possible after acceptance. "Just Accepted" manuscripts appear in full in PDF format accompanied by an HTML abstract. "Just Accepted" manuscripts have been fully peer reviewed, but should not be considered the official version of record. They are citable by the Digital Object Identifier (DOI®). "Just Accepted" is an optional service offered to authors. Therefore, the "Just Accepted" Web site may not include all articles that will be published in the journal. After a manuscript is technically edited and formatted, it will be removed from the "Just Accepted" Web site and published as an ASAP article. Note that technical editing may introduce minor changes to the manuscript text and/or graphics which could affect content, and all legal disclaimers and ethical guidelines that apply to the journal pertain. ACS cannot be held responsible for errors or consequences arising from the use of information contained in these "Just Accepted" manuscripts. 


\title{
Controlling the self-assembly behavior of aqueous
}

\section{chitin nanocrystal suspensions}

\author{
Aurimas Narkevicius, Lisa M. Steiner, Richard M. Parker, Yu \\ Ogawat, Bruno Frka-Petesic, Silvia Vignolini* \\ Department of Chemistry, University of Cambridge, Lensfield \\ Road, Cambridge CB2 1EW, United Kingdom
}

\begin{abstract}
As with many other bio-sourced colloids, chitin nanocrystals (ChNCs) can form liquid crystalline phases with chiral nematic ordering. In this work, we demonstrate that it is possible to finely tune the liquid crystalline behavior of aqueous chNC suspensions. Such control was made possible by carefully studying how the hydrolysis conditions and suspension treatments affect the colloidal and self-assembly properties of ChNCs. Specifically, we systematically investigate the effects of duration and acidity of chitin hydrolysis required to extract ChNCs, as well as the effects of the tip sonication energy input, degree of acetylation, pH and ionic strength. Finally, we show that by controlled water evaporation, it is possible to retain and control the helicoidal ordering in dry films, leading


to a hierarchical architecture analogous to that found in nature, e.g. in crab shells. We believe that this work serves as a comprehensive insight into ChNC preparation and handling which is required to unlock the full potential of this material in both a scientific and industrial context.

\section{INTRODUCTION}

Lyotropic liquid crystalline phases have been observed for many biopolymers, from DNA and proteins to polysaccharides. ${ }^{1}$ In nature, these biological building blocks appear to directly encode the functions and properties of incredibly diverse hierarchical materials. As such, the ability to artificially control such building blocks and their interactions at the nanoscale is highly desirable for the fabrication of sustainable functional materials.

This is especially true in the case of the two most abundant biopolymers available on the planet, cellulose and chitin. In their colloidal form, they both spontaneously self-organize into a chiral nematic liquid crystalline phase.2,3 However, while the self-assembly of colloidal cellulose (a.k.a. cellulose nanocrystals) has attracted significant attention in recent years, 2,4-6 liquid crystalline phases of chitin nanocrystals (ChNCs) remain virtually unexplored. In fact, despite the pioneering works by Revol et $a l^{3}$, Li et $a l^{7}$ and Belamie et $a l^{8}$ 
that revealed the lyotropic behavior of ChNC in water, a systematic assessment on the impact of each of the preparation steps or post-treatments upon the ChNC suspension has yet to be reported. Here, by carefully addressing all the steps required to prepare an aqueous ChNC suspension; from initial hydrolysis of chitin, to controlling the degree of acetylation, the extent of tip sonication and the ionic strength or $\mathrm{pH}$, we demonstrate how to optimise the stability of the ChNC suspension, control its self-organisation into a chiral nematic liquid crystal and ultimately produce well-ordered helicoidal films with a defined pitch, tuneable from a few hundred nanometres to several microns.

As a natural linear polysaccharide, chitin is an extremely abundant biomaterial and is found in crustacean, insect, and beetle exoskeletons, as well as the cell walls of fungi (Figure 1A).9 In nature, chitin is structured into fibers that are comprised of bundles of semi-crystalline microfibrils. Each microfibril is composed of extended linear molecular chains of an acetylglucosamine homopolymer with only the surface having free amines (Figure 1B). ${ }^{9}$ Chitin microfibrils are used for mechanical support in many living organisms, often in the form of a finely-tuned helicoidal structure that serves to significantly increase the mechanical robustness of e.g. crab exoskeletons. ${ }^{10}$ 
A Prawn shells

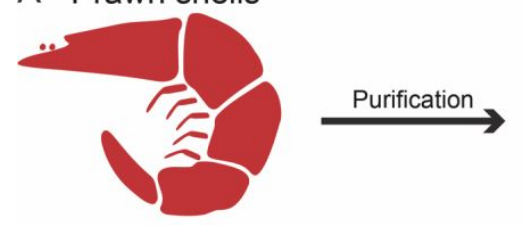

E Solid free standing films

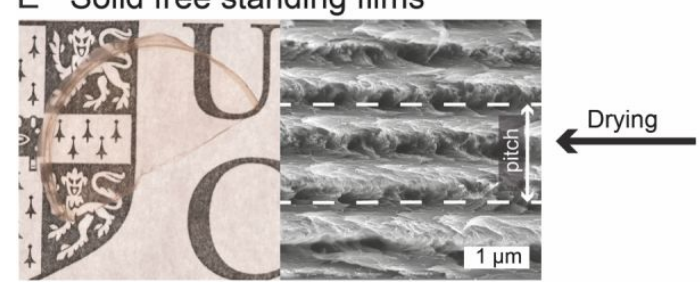

B Chitin microfibers

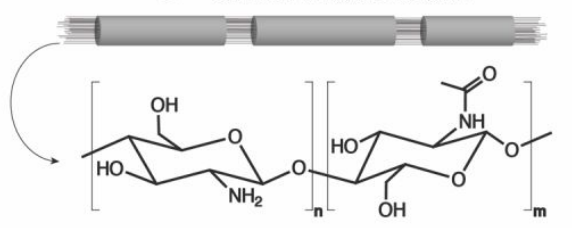

D Chiral nematic phase

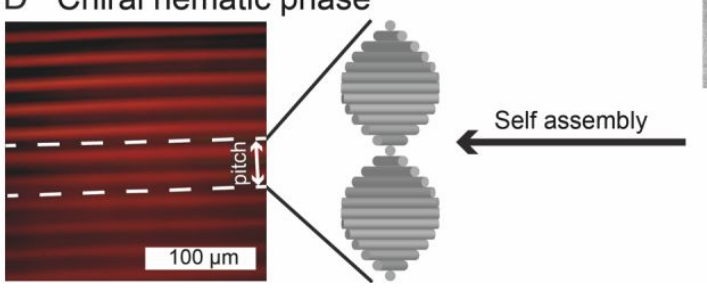

C Chitin nanocrystals
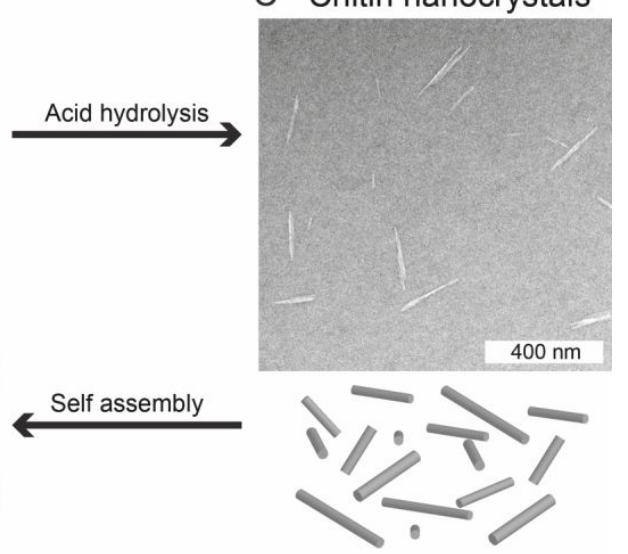

Figure 1. Overview of chitin nanocrystal preparation and the subsequent self-assembly into helicoidal films: Prawn shell chitin powder (A) is purified to yield chitin microfibers (B), that upon acid hydrolysis form chitin nanocrystals (C). Under controlled conditions, colloidal chitin nanocrystal suspension (C) can selfassemble into a chiral nematic phase (D), that upon evaporation yields a transparent film that retains this helicoidal nanoarchitecture (E). The logo is used with permission from University of Cambridge.

ChNCs can be isolated from purified natural chitin by strong acid hydrolysis. ${ }^{11}$ The resultant rod-shaped nanoparticles are highly polydisperse and their dimensions vary between 50 $300 \mathrm{~nm}$ in length and $6-8 \mathrm{~nm}$ in width (Figure 1C). ${ }^{3}$ In water, ChNCs carry a positive surface charge, which originates from protonated amines at the ChNC surface, with an average pKa value of $~ 6.3 .12$ At a pH below this pKa, i.e. acidic aqueous media, ChNCs are colloidally stable. Similarly to cellulose 
nanocrystals, ${ }^{2}$ colloidal ChNCs exhibit liquid crystalline behavior above a threshold concentration. ${ }^{3,8}$ This is an entropy driven process dictated by the anisotropic shape and the mutual interactions between the charged, rod-like ChNCs. ${ }^{13-16}$ This selfassembly process starts by a nucleation and growth mechanism until a macroscopic phase separation occurs. ${ }^{2,3}$ The anisotropic ChNC phase has a chiral nematic order, as identified by the characteristic 'fingerprint pattern' in polarized optical microscopy (Figure 1D).3,8 In this structure, ChNCs locally align along a direction that spatially rotates in a left-handed fashion about an axis. ${ }^{17}$ The helix achieves a $360^{\circ}$ rotation over the distance which is defined as the chiral nematic pitch and corresponds to twice the periodicity of the measured fingerprint pattern. Upon evaporation this ordering can be retained giving rise to films with a defined helicoidal nanoarchitecture (Figure 1E).

\section{EXPERIMENTAL METHODS}

All reagents, unless stated otherwise, were acquired from Sigma Aldrich. Chitin (from shrimp shells, practical grade, powder) was purified by sodium hydroxide treatment ( 5 wto, $80{ }^{\circ} \mathrm{C}, 3 \mathrm{~h}$ ), followed by acidic bleaching in sodium chlorite $\left(0.3\right.$ wt\%, $80{ }^{\circ} \mathrm{C}$, 3 h). ${ }^{18}$ These purification steps were repeated three times each before dialyzing against Milli-Q water (Regenerated Cellulose 
dialysis tubing, MWCO 12-14 kDa), and subsequent isolation by centrifugation to form a pellet $\left(25,000 \mathrm{~g}, 4{ }^{\circ} \mathrm{C}, 30 \mathrm{~min}\right)$, as described in detail in the ESI.

The purified chitin pellet ( 10 wto chitin in water) was hydrolyzed in hydrochloric acid ( $\mathrm{HCl}, 3.0-5.0 \mathrm{M}$ ) under reflux (104 ${ }^{\circ} \mathrm{C}, \quad 90-540 \mathrm{~min}$, Table 1) as adapted from previously reported protocols.3,18,19 The reaction mixture was quenched by diluting with ice-cold Milli-Q water and washed twice by centrifugation (as above). The produced ChNC suspension was then dialyzed against Milli-Q water until the conductivity of the surrounding bath remained stable for two days. The ChNC concentration was measured by drying three samples at $45{ }^{\circ} \mathrm{C}$ ( $1 \mathrm{~g}$ suspension) and measuring the dry content. The ChNC suspensions were diluted to 1.0 wto and dialyzed against a dilute $\mathrm{HCl}$ solution (1.0 mM) until stable at $\mathrm{pH} 3.0$ for two days. ChNCs were then dispersed using tip sonication. The samples were subsequently vacuum filtered through nitrocellulose filter paper with pore sizes of $8.0 \mu \mathrm{m}$ and $0.8 \mu \mathrm{m}$. The final HCl and $\mathrm{NaCl}$ concentration was adjusted by a further dialysis, followed by concentration using rotary evaporation. Thus, all the samples in this study have a fixed electrolyte ratio of 1.0 mM HCl to 1.0 wto ChNC, except where otherwise stated. For example, at 1.0 wto $\mathrm{ChNC}$ the $\mathrm{HCl}$ concentration is $1.0 \mathrm{mM}$, while at 10.0 wto the $\mathrm{HCl}$ concentration is $10.0 \mathrm{mM}$. At 1.0 wto ChNC 
the $\mathrm{pH}$ is 3.0 and decreases upon increasing ChNC concentration, reaching $\mathrm{pH} 2.0$ at 10.0 wto of ChNC. Other ionic strengths were prepared in an analogous fashion, as described in detail in the ESI.

Chitin and ChNC were analyzed for their chemical composition by freeze-drying samples, followed by FT-IR spectroscopy, solidstate ${ }^{13} \mathrm{C}$ CP-MAS NMR spectroscopy (sSNMR) and PXRD analysis. All characterization methods showed that purification has been successful and that ChNC are chemically equivalent to chitin, as described in detail in the ESI.

ChNC suspensions were assessed for their colloidal properties by means of dynamic light scattering (DLS), ろ-potential, transmission electron microscopy (TEM), UV-Vis spectroscopy and conductometric titration, as described in detail in the ESI.

ChNC suspensions were concentrated using a rotary evaporator at $55^{\circ} \mathrm{C}$ until a viscous fluid was attained (7-13 wto). To assess liquid crystalline behavior, ChNC concentration series were produced through dilution with Milli-Q water. Phase separation was then quantified by sealing the series of ChNC suspensions into round capillaries (inner diameter $\approx 1.3 \mathrm{~mm}$ ) and, after storing vertically for at least a month to ensure equilibration. Photographs between crossed polarizers were taken and the ratio between the height of the bright anisotropic phase to the whole suspension was defined as the volume fraction of the anisotropic 
phase. Pitch measurements were performed by placing capillaries horizontally under a cross-polarized optical microscope in transmission mode (SLWD objective, 20x magnification), as described in detail in the ESI.

\section{RESULTS AND DISCUSSION}

Hydrolysis conditions. The physical properties of ChNCs produced from chitin hydrolysis depend on key reaction conditions, such as duration and acidity (Figure 2). Therefore, the effect of the hydrolysis time was examined by comparing ChNC samples hydrolyzed for 90, 270 and 540 minutes in boiling hydrochloric acid ( $\mathrm{HCl}, 3.0 \mathrm{M})$. These samples are referred to as 3M90, 3M270 and 3M540, respectively. Similarly, the effect of increased acidity was screened with $5.0 \mathrm{M} \mathrm{HCl}$ and are likewise referred to as 5M90, 5M180, and 5M270. For consistency, an equivalent tip sonication treatment of $20 \mathrm{~kJ} / \mathrm{g}_{\mathrm{ChNC}}$ was applied to all the samples and the ionic strength was standardized to $100 \mu \mathrm{mol} / \mathrm{g}_{\mathrm{ChNC}}$ by dialysis against $\mathrm{HCl}$.

Figure 2A-C and Table 1 show that the hydrolysis time markedly affects ChNC dimensions and their colloidal stability. 3M90 results in notably larger particles with significantly higher polydispersity compared to the other samples. Therefore, it can be concluded that 3 M90 conditions are insufficient to hydrolyze 

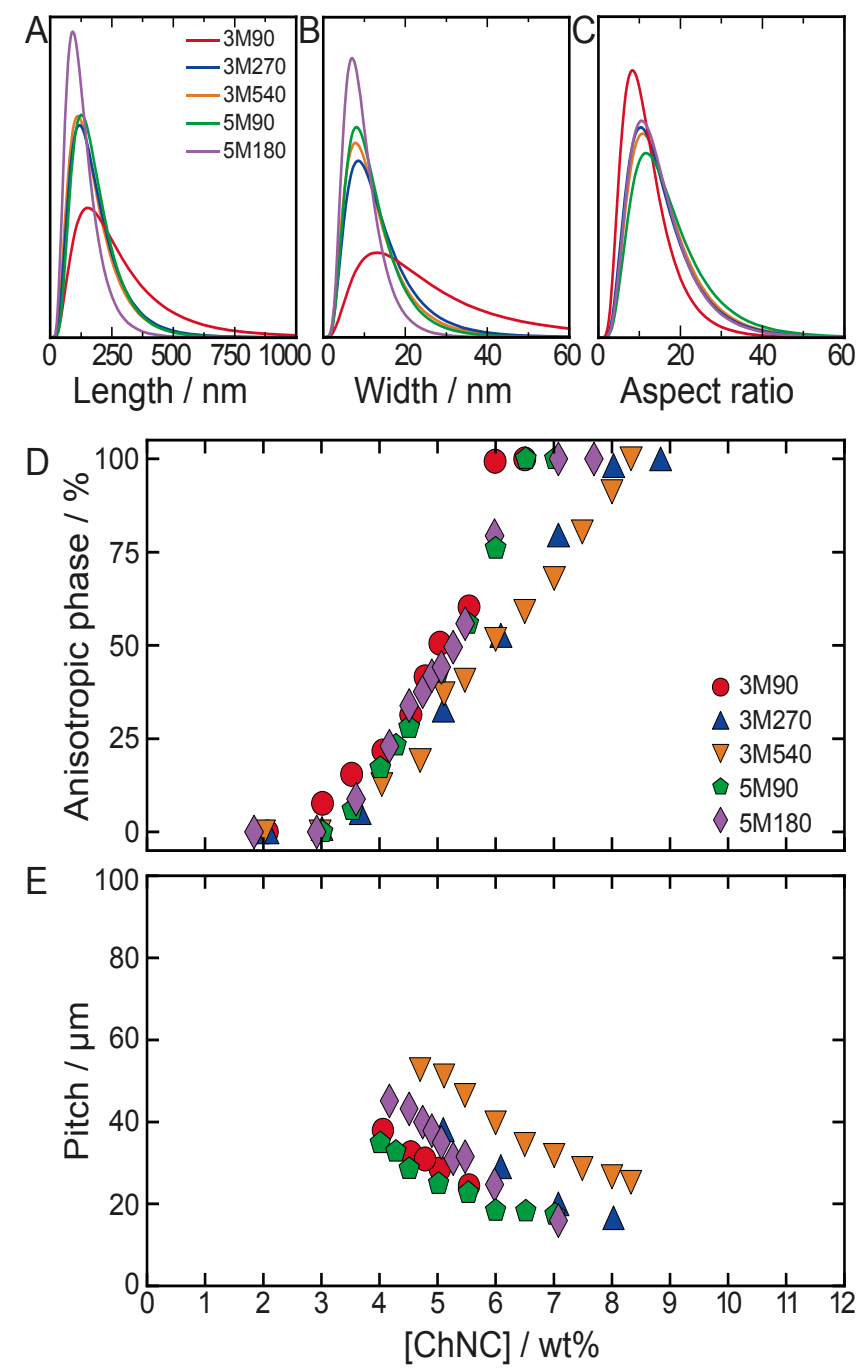

Figure 2. Effect of hydrolysis conditions on ChNC dimensions and self-assembly. (A-C) The fitted lognormal distribution curves from TEM measurements of ChNC (A) length, (B) width and (C) aspect ratio (Figure $\mathrm{S9}$ provides full histograms). Plots showing (D) the increasing proportion of anisotropic phase and decreasing chiral nematic pitch upon increasing ChNC concentration. 
chitin so as to achieve a well-dispersed stable ChNC suspension. These results agree with the lowest surface charge evaluated by conductometric titration and $\zeta$-potential values of the resulting suspension (Table 1). In contrast, ChNC suspensions prepared at 3M270 and 3M540 displayed excellent colloidal stability. Both ChNC suspensions remained sediment free over several weeks and their turbidity was markedly lower when compared to 3M90 (Figure S1A), indicating both smaller ChNCs and a notable absence of aggregates. The slight decrease in turbidity, Z-average particle size (as measured by DLS) and an increase in ל-potential and surface charge when 3M270 is compared to 3M540 are consistent with prolonged acid hydrolysis slowly degrading the crystalline part (Table 1). Moreover, the length and width distributions of 3M270 and 3M540 overlap (Figure 2A,B), thus it was concluded that chitin is already sufficiently hydrolyzed within 270 minutes to achieve a stable and well-dispersed ChNC suspension ( Figure 2A, B) .

The effect of acidity on the hydrolysis process was also evaluated. An increase in $\mathrm{HCl}$ concentration was found to simply accelerate chitin hydrolysis and as a result ChNC suspensions prepared at 5M270 were found to be dark grey/brown in appearance (i.e. over hydrolyzed). As this is indicative of decomposition processes, it was not studied further. 5M180, on the other hand, displayed the smallest ChNC dimensions and lowest polydispersity 
of all ChNC samples prepared in this study (Figure 2A, B; Figure S9). Despite the DLS measurements indicating it has almost identical Z-average size and turbidity as compared to 3M540 (indicating similar degree of chitin hydrolysis), the 5M180 ChNC suspension exhibits a lower ChNC surface charge and ל-potential (Figure S1A; Table 1). ChNC following 5M90 preparation conditions were of dimensions similar to 3M270, but again with a much smaller surface charge (Figure 2; Table 1). Overall, it implies that increasing hydrochloric acid concentration from 3.0 to $5.0 \mathrm{M}$ accelerated chitin hydrolysis at the glycosidic bond while having little effect on the hydrolysis of surface amide groups. This observation is consistent with reports showing that the amide hydrolysis rate plateaus at similarly high hydrochloric acid concentrations.20,21 Thus, the hydrolysis duration is the main parameter controlling the maximum ChNC surface charge.

The liquid crystalline behavior was analyzed by phase diagrams obtained Via serial dilution of each concentrated ChNC suspension, allowing comparison of the proportion of the liquid crystalline phase formed as a function of ChNC concentration. It was observed that all ChNC suspensions, including 3M90, macroscopically phase separated with increasing ChNC concentration (Figure 2D) and gave rise to a chiral nematic 
phase, as quantified by polarized optical microscopy ( Figure 2E)

In order to discuss the variations observed between these samples, we first introduce some scaling arguments based on Onsager's theory of soft repulsing rods.13,22 The concentration at which the ChNC suspension separates into a biphasic $\left(c_{I}\right)$ and then in a fully chiral nematic phase $\left(c_{A}\right)$ can be correlated to the aspect ratios $(L / D)$ of the ChNCs and also to their mutual interactions. ${ }^{14}$ To account for the latter, we can consider that each ChNC occupies an effective cylindrical volume that cannot overlap with other ChNCs, leading to an effective diameter $D_{\text {eff }}$ and length $L_{\text {eff }}$. These effective dimensions, $D_{\text {eff }}$ and $L_{\text {eff }}$ are expected to depend both on the thickness of the ionic doublelayer surrounding the ChNCs (scaling as the Debye length $\kappa^{-1}$ and controlled by the ionic strength as $\kappa^{-1}(\mathrm{~nm})=0.304 / \sqrt{\mathrm{I}(\mathrm{M})}$ in aqueous medium) and the surface charge of the ChNCs (in a non-linear fashion) $\cdot^{14}$

This leads to an effective volume and thus concentration $c_{\text {eff }}$ but also aspect ratio $L_{\text {eff }} / D_{\text {eff }}$. As a result, if the effective threshold concentrations scale as $c_{j, \text { eff }} \propto D_{\text {eff }} / L_{\text {eff }}$, the actual concentration $c_{j}$ should scale as:

$$
c_{j} \propto c_{j, \mathrm{eff}}\left(\frac{V}{V_{\text {eff }}}\right) \propto c_{j, \mathrm{eff}} \frac{D^{2} L}{D_{\mathrm{eff}}^{2} L_{\mathrm{eff}}} \propto \frac{D_{\text {eff }} D^{2} L}{L_{\mathrm{eff}} D_{\mathrm{eff}}^{2} L_{\mathrm{eff}}} \propto \frac{D^{2} L}{D_{\mathrm{eff}} L_{\mathrm{eff}}^{2}}
$$


Since the relative length variation is negligible compared to the relative diameter variation $\left(L_{\mathrm{eff}} \sim L\right)$, this can be simplified to:

$$
c_{j} \propto \frac{D^{2}}{D_{\text {eff }}}=\frac{D}{D_{\text {eff }}}\left(\frac{D}{L}\right)
$$

where the largest variations of $c_{j}$ are expected for ChNCs of small aspect ratios. In a polydisperse suspension, rods of larger aspect ratios turn chiral nematic at a lower concentration and thus are mostly responsible of the observed $c_{I}$, while the rods of smallest aspect ratios are expected to turn chiral nematic at higher concentration, thus impacting mostly $\boldsymbol{c}_{A}$. A direct consequence of this is that a variation in the interactions between rods would cause more variation of the $c_{A}$ value (for which the associated rods have a smaller aspect ratio) than the $c_{I}$ value. ${ }^{15}$

The observation of the phase diagrams in Figure 2D is in good agreement with this description. The phase separation starts at a similar ChNC concentration $c_{I} \sim 3$ wto for all samples (Figure 2C,D), which suggests that ChNCs with a high aspect ratio (i.e. long and thin) are present in all the samples. In contrast, the second threshold concentration, $c_{A}$, varies much more between the samples and increases with the hydrolysis time. This correlates with the presence of shorter rods and agrees with the expected 
trend, however, its interpretation might also involve differences in the ChNC colloidal stability.

3M90 exhibited the lowest $c_{A}$ and $c_{I}$ concentration (Figure 2D; Table 1), in agreement with a smaller proportion of short ChNCs and overall lower colloidal stability. Closer inspection into this sample revealed a sharp increase in the volume of the anisotropic phase at 6 wto of ChNC, while at lower concentrations a near linear relationship was observed. This suggests an interruption of the macroscopic separation of the two phases and can further be verified from the incomplete phase separation just below this concentration (Figure S6, S7). The lack of relaxation over time implied that a slow gelation occurred in the suspension. ${ }^{23-25}$ Other samples in the series did not exhibit such behavior due to higher colloidal stability. 3M270 and 3M540 both had high $c_{A}$ thresholds in comparison with the 5M90 and 5M180, and also more amine groups and thus a higher structural charge when protonated (Figure 2D, Table 1).

The pitch, in general, decreased with ChNC concentration (Figure 2E) and is a consequence of ChNCs getting in closer proximity with each other. Thus, they interact and twist more with respect to each other, resulting in a lower chiral nematic pitch. In addition, we relate the increase in pitch to the surface charge, as exemplified by 3M540, where the highest surface charge results in the largest $D_{\text {eff }}$ and as such the largest 
pitch. However, to date no theory accounts for polydisperse suspensions or the pitch evolution when chiral rods are considered.

We conclude that 3M270, 3M540, 5M90 and 5M180 prove to be appropriate conditions to prepare stable self-assembling colloidal ChNC suspensions, with 3M270 and 3M540 having a higher surface charge. The use of $5.0 \mathrm{M} \mathrm{HCl}$ also corresponds to much harsher reaction conditions, which are also harder to control and less environmentally-friendly. For these reasons, $3.0 \mathrm{M} \mathrm{HCl}$ was preferred in the following studies. More specifically, the 3M540 hydrolysis condition was chosen to test the effects of the other parameters as these conditions yielded ChNC suspensions with slightly smaller dimensions and higher surface charge, as compared to $3 \mathrm{M} 270$.

Tip sonication. Tip sonication is a common post hydrolysis treatment that breaks apart large bundles of chitin crystallites, resulting in well-dispersed ChNC suspensions. For that reason, the application of tip sonication can have an important impact on the final properties of the suspension and should not be neglected.

The influence of tip sonication energy input was studied by preparing a new batch of 3M540 ChNC suspension. Three different tip sonication conditions were then tested, namely, Ts4, Ts20, 

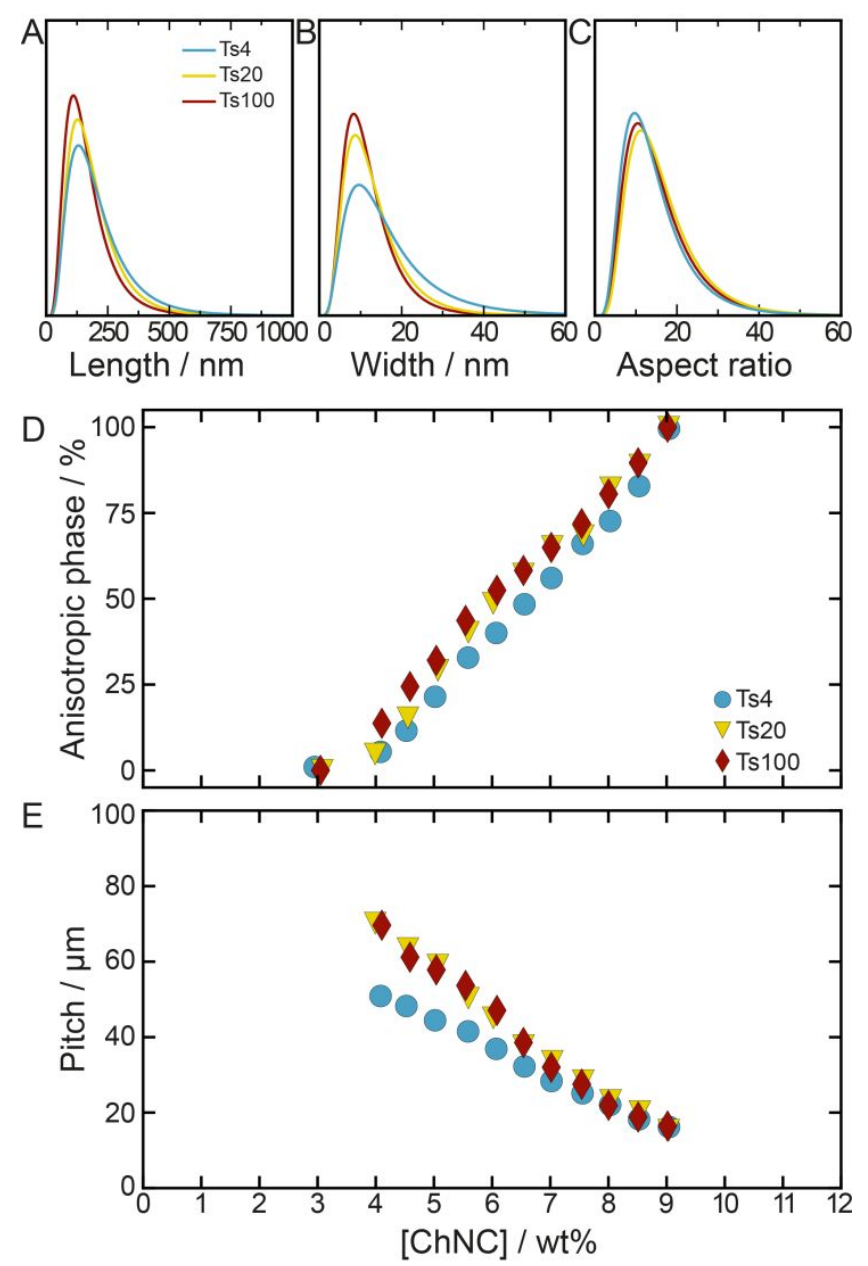

Figure 3. Effect of increasing tip sonication energy input on ChNC dimensions and self-assembly. (A-C) The fitted lognormal distribution curves from TEM measurements of ChNC (A) length, (B) width and (C) aspect ratio (Figure sio provides full histograms). Plots showing (D) the increasing proportion of anisotropic phase and (E) decreasing chiral nematic pitch with increasing ChNC concentration. 
and Ts100 referring to the energy inputs of 4, 20, and $100 \mathrm{~kJ} / g_{\text {chnc }}$ respectively. Other conditions were kept constant, including the ionic strength and acidity, which were standardized to $100 \mu \mathrm{mol} / \mathrm{g}_{\mathrm{ChNC}}$ by dialysis against $\mathrm{HCl}$.

As expected, we observed that tip sonication treatment reduced the dimensions and polydispersity of ChNCs (Figure 3A-C; Figure S10). The decrease in turbidity (Figure S1B) and Zaverage size (Table 1) both provide a robust confirmation that the average ChNC dimensions decreased with tip sonication energy. More precisely, TEM measurements indicate that Ts4 has both the longest and thickest ChNCs when compared to Ts20 and Ts100 (see $L, D$ and $L \times D$ in Figure S10, S12 and Table S2). The latter two samples are very similar except that Tsio0 has a slightly lower polydispersity as seen from the shorter tails in the length and width distributions in the TEM histograms. Tip sonication, as expected, had no effect on the surface charge as measured by conductometric titration (Table 1).

The phase diagrams of these ChNC suspensions are presented in Figure 3D. The first and second threshold concentrations, $\boldsymbol{c}_{I}$ and $c_{A}$, are comparable for all three samples, in agreement with little variations in the aspect ratio and the surface charge (Figure 3C; Table 1). However, the chiral nematic pitch varies more significantly (Figure 3E) - samples Ts20 and Ts100 present a larger pitch at lower ChNC concentrations and, importantly, 
show a steeper decrease in pitch with increasing chNC concentration when compared to Ts4. The suspensions were dialyzed together after the tip sonication treatment to rule out the potential effect of ionic strength difference, which was indicated for a similar system of cellulose nanocrystals. ${ }^{26}$ A more likely explanation would be that tip sonication breaks apart ChNC bundles with a strong chiral shape, the presence of which would provide a higher twisting power in the anisotropic suspension. As such, breaking these "colloidal chiral dopants" into smaller rod-like units should lead to a smaller twist between ChNCs and thus a larger pitch. ${ }^{2}$

Notably, the sample prior to any tip sonication treatment did not display any sign of poor stability (no sedimentation occurred), however, the suspension appeared white due to the scattering of larger bundles. As such tip sonication was always applied to consistently disperse the particles prior to studying the liquid crystalline behavior of the system. This indicates that the tip sonication treatment investigated here is primarily breaking already colloidally stable ChNCs into smaller units, and that most of the loose bundles were already broken apart under Ts20, while Ts100 provided only marginal changes. However, Ts100 was chosen for further investigations as it results in slightly lower polydispersity (i.e. shorter tail in TEM 
Table 1. A summary of the conditions used to prepare ChNC suspensions and their resultant colloidal properties.

\begin{tabular}{|c|c|c|c|c|c|c|c|c|c|}
\hline & $\begin{array}{l}{[\mathrm{HCl}} \\
]_{\mathrm{M}} /\end{array}$ & $\begin{array}{l}\text { Tim } \\
\text { e / } \\
\text { min }\end{array}$ & $\begin{array}{l}\text { Tip } \\
\text { sonicati } \\
\text { on } \\
\text { energy }\end{array}$ & $\begin{array}{l}\text { TEM } \\
\text { leng } \\
\text { th } \\
\text { mean } \\
/ \mathrm{nm}\end{array}$ & $\begin{array}{l}\text { TEM } \\
\text { widt } \\
\text { h } \\
\text { mean } \\
/ \mathrm{nm}\end{array}$ & $\begin{array}{l}\text { Z- } \\
\text { avera } \\
\text { ge / } \\
\text { nm }\end{array}$ & $\begin{array}{l}\zeta- \\
\text { potenti } \\
\text { al / mV }\end{array}$ & $\begin{array}{l}n\left(\mathrm{NH}_{2}\right. \\
)^{\mathrm{b}}\end{array}$ & $\begin{array}{l}\text { Yiel } \\
\text { d / } \\
\text { o }\end{array}$ \\
\hline $3 \mathrm{M} 90$ & 3 & 90 & 20 & $\begin{array}{l}286 \\
\pm \\
192\end{array}$ & $\begin{array}{ll}28 & \pm \\
23 & \end{array}$ & $\begin{array}{ll}277 & \pm \\
112\end{array}$ & $39 \pm 5$ & 120 & 88 \\
\hline $\begin{array}{l}3 \mathrm{M} 27 \\
0\end{array}$ & 3 & 270 & 20 & $\begin{array}{l}184 \\
\pm \quad 98\end{array}$ & $\begin{array}{ll}14 \pm \\
9\end{array}$ & $\begin{array}{ll}237 & \pm \\
101\end{array}$ & $45 \pm 9$ & 233 & 79 \\
\hline $\begin{array}{l}3 \mathrm{M} 54 \\
\mathrm{O}^{+}\end{array}$ & 3 & 540 & 20 & $\begin{array}{l}176 \\
\pm \quad 95\end{array}$ & $\begin{array}{ll}12 \pm \\
8\end{array}$ & $\begin{array}{l}210 \pm \\
85\end{array}$ & $55 \pm 9$ & 248 & 70 \\
\hline $5 \mathrm{M} 90$ & 5 & 90 & 20 & $\begin{array}{l}182 \\
\pm \quad 87\end{array}$ & $\begin{array}{ll}13 & \pm \\
14 & \end{array}$ & $\begin{array}{l}241 \quad \pm \\
95\end{array}$ & $50 \pm 7$ & 144 & 75 \\
\hline $\begin{array}{l}5 \mathrm{M} 18 \\
0\end{array}$ & 5 & 180 & 20 & $\begin{array}{l}133 \\
\pm \quad 66\end{array}$ & $\begin{array}{ll}10 \pm \\
5\end{array}$ & $\begin{array}{l}212 \pm \\
94\end{array}$ & $48 \pm 14$ & 182 & 65 \\
\hline Ts 4 & 3 & 540 & 4 & $\begin{array}{l}211 \\
\pm \\
131\end{array}$ & $\begin{array}{l}18 \pm \\
14\end{array}$ & $\begin{array}{l}291 \\
115\end{array}$ & $54 \pm 12$ & 248 & - \\
\hline $\begin{array}{l}\text { Ts20 } \\
+\end{array}$ & 3 & 540 & 20 & $\begin{array}{l}189 \\
\pm \quad 99\end{array}$ & $\begin{array}{ll}13 \pm \\
8\end{array}$ & $\begin{array}{l}195 \quad \pm \\
115\end{array}$ & $56 \pm 12$ & 246 & - \\
\hline $\begin{array}{l}\text { Ts10 } \\
0^{\neq}\end{array}$ & 3 & 540 & 100 & $\begin{array}{l}166 \\
\pm \quad 88\end{array}$ & $\begin{array}{l}12 \pm \\
7\end{array}$ & $\begin{array}{l}175 \pm \\
67\end{array}$ & $59 \pm 13$ & 247 & - \\
\hline$A C^{\ddagger}$ & 3 & 540 & 100 & $\begin{array}{l}178 \\
+\quad 88\end{array}$ & $\begin{array}{ll}18 \pm \\
7 & \end{array}$ & $\begin{array}{l}173 \quad \pm \\
73\end{array}$ & $57 \pm 13$ & 269 & - \\
\hline deAc & 3 & 540 & 100 & $\begin{array}{l}153 \\
\pm \quad 78\end{array}$ & $\begin{array}{l}12 \pm \\
6\end{array}$ & $\begin{array}{l}137 \quad \pm \\
58\end{array}$ & $56 \pm 10$ & 289 & \\
\hline
\end{tabular}

a Expressed in $\mathrm{kJ}$ per $\mathrm{g}_{\mathrm{ChNC}}$

b Expressed in mmol $\left(\mathrm{NH}_{2}\right)$ per $\mathrm{kg}_{\mathrm{ChNC}}$ 
Samples produced using comparable conditions are paired with ${ }^{\dagger}$ and ${ }^{\ddagger}$ symbols (column 1 ).

distribution data, Figure 3A,B; Table 1). It should also be noted that the amount of tip sonication energy required to achieve results analogous to Ts20 might differ depending on the hydrolysis conditions.

Degree of acetylation. Chitin is commonly treated with a strong base, such as sodium hydroxide, to obtain chitosan - a fully deacetylated form of chitin. ${ }^{27}$ A controlled treatment can also lead to a desired degree of acetylation (DA), which is defined as the percentage of amide groups with respect to sum of all the amine and amide groups. Thus, deacetylation offers a route to control the potential maximum surface charge on ChNC and thus their self-assembly behavior. In fact, it has been previously reported that the DA of ChNC decreases when they are prepared from deacetylated chitin. ${ }^{7}$ However, contrary to this finding, we observed that the surface charge of ChNC prepared from deacetylated chitin is rather unaffected and the differences in ChNC self-assembly behavior arise instead from changes in ChNC dimensions - a point which was not addressed in the previous work. ${ }^{7}$ 
The effects of the degree of acetylation were studied by preparing two chitin samples: purified chitin (Ac-Chitin) and deacetylated purified chitin (dAc-Chitin). The degree of acetylation and surface charge were assessed by three methods: solid-state NMR spectroscopy (ssNMR), which measures both the core and the surface of the chitin nanocrystals, as well as conductometric titration and ל-potential measurements which probe only the surface of the chitin nanocrystal. ssNMR indicated that the deacetylation of purified chitin was successful, with DA decreasing from 97\% (Ac-Chitin) to $89 \%$ (deAc-Chitin). However, the produced Ac-ChNC and deAc-ChNC both had $\mathrm{a} D A \approx 98 \%$ after hydrolysis, similar to that recorded for

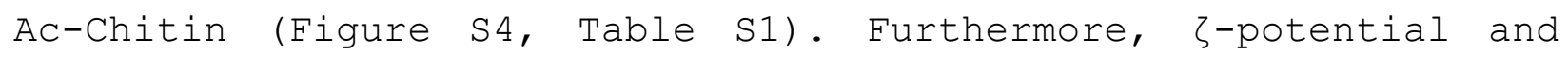
conductometric titration measurements support the fact that deAc-ChNC and Ac-ChNC have almost identical surface charge (Table 1).

Chitin is insoluble in aqueous media and thus the surface of the microfibers is readily available for deacetylation, whereas the core would be largely unaffected. However, deacetylated chitin is much more soluble in the acidic conditions present during hydrolysis, allowing the deacetylated surface to be easily removed, which would reduce the widths of the resulting ChNCs. In contrast, the same treatment on less soluble Ac-Chitin 

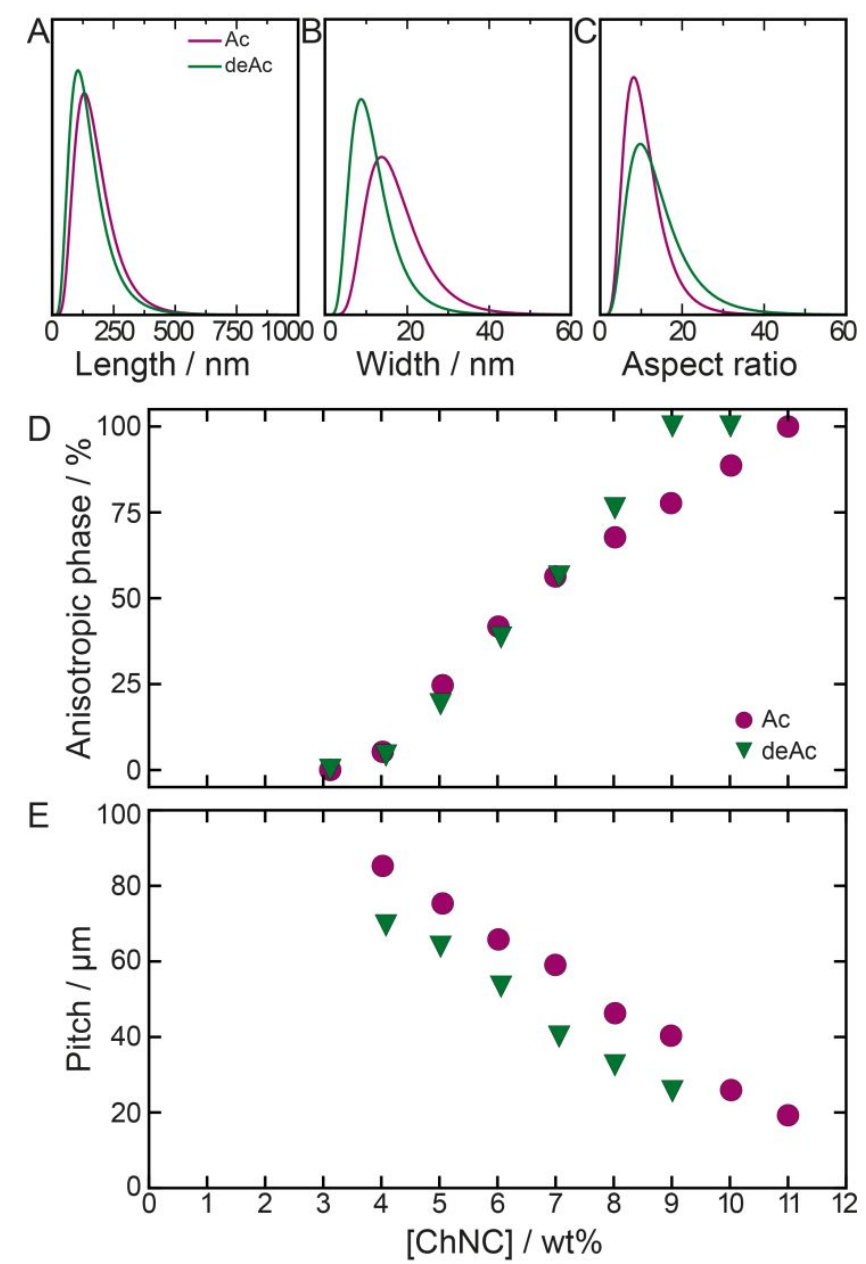

Figure 4. Effect of degree of acetylation on ChNC dimensions and self-assembly. (A-C) The fitted lognormal distribution curves from TEM measurements of ChNC (A) length, (B) width and (C) aspect ratio (Figure S11 provides full histograms). Plots showing (D) the increasing proportion of anisotropic phase and (E) decreasing chiral nematic pitch upon increasing ChNC concentration. 
would not lead to an identical etching. The surface charge on both ChNC suspensions was found to be almost equivalent, suggesting that most of the surface which was affected by sodium hydroxide deacetylation, as in deAc-Chitin, was exfoliated during the hydrolysis step. Thus, the apparent increase in the DA of deAc-ChNC compared to deAc-Chitin is not because of "reacetylation" but because of the "etching" of the deacetylated surface during hydrolysis. Indeed, length and width histograms from TEM analysis show that deAc-ChNC are shorter and thinner than the Ac-ChNC and have a higher aspect ratio (Figure 4A-C; Figure S11). This hypothesis is also supported by the DLS Zaverage size (Table 1) and turbidity values (Figure S1C), indicating smaller ChNCs in the deAc-ChNC sample when compared to Ac-ChNC. The changes in the aspect ratio influence chNC selfassembly, which results in deAc-ChNC reaching a fully anisotropic phase at a lower concentration than Ac-ChNC (Figure 4D). Additionally, the pitch is only slightly smaller for the deAc-ChNC suspension and it comparably decreases with ChNC concentration for both suspensions. These observations are consistent with the surface charge being effectively comparable and the aspect ratio of deAc-ChNC being larger (Figure 4E).

Chitin deacetylation prior to hydrolysis thus provides a way to increase ChNC aspect ratio without a significant change in the surface charge. This characteristic can potentially be repeated 
to obtain much thinner crystals and is unique to chitin, whereas the physical aspect ratio of cellulose nanocrystals, as a comparison, can only be addressed by changing the source and the hydrolysis conditions, which provides less flexibility. ${ }^{28}$

Ionic strength and $\mathbf{p H}$. The ChNC surface charge can be modulated by controlling the $\mathrm{pH}$. The surface-bound amine groups become increasingly protonated at $\mathrm{pH}$ values below their pKa value. ${ }^{29}$ Therefore, at lower pH, the surface charge is higher, which favors repulsive interactions and thus a larger effective diameter $D_{\text {eff }}$. However, reducing $\mathrm{pH}$ causes an increase in the ionic strength of the medium, and consequently decreases the Debye length, which favors a smaller $D_{\text {eff }}$. Therefore, the degree of electrostatic repulsion between ChNCs is dependent on both the explicit acid concentration and the overall ionic strength, which produce opposite effects. Both parameters have to be carefuly considered to control their self-assembly while maintaining the colloidally stability of the system. ${ }^{8,30}$

For the study on the interplay between acidity and ionic strength on the self-assembly of ChNC, a 3M540 Tsion ChNC suspension was used. Different ionic strengths and acid concentrations were achieved by dialysis against $\mathrm{NaCl}$ and $\mathrm{HCl}$ solutions followed by a concentration step using a rotary evaporator. Therefore, the ratio between the electrolyte and 
ChNC concentrations was maintained which would mimic the chNC suspension behavior upon drying .

The effects of $\mathrm{HCl}$ concentration and total ionic strength can be partly decoupled by preparing a series of ChNC suspensions with increasing $\mathrm{HCl}$ concentration while adjusting the total ionic strength to be equivalent with NaCl. As such, three samples were prepared with increasing HCl concentration of 1, 10 and $100 \mu \mathrm{mol} / \mathrm{g}_{\mathrm{chNc}}$, but an equivalent total ionic strength of 100 umol/g $g_{\text {chnc }}$. Therefore, this experimental design allows an increase ChNC surface charge when $\mathrm{HCl}$ concentration is raised, resulting in a larger effective diameter $D_{\text {eff }}$. As a consequence, the effective aspect ratio is reduced, and a higher threshold concentration is expected. This agrees well with the observations of the second threshold concentration shifting to higher ChNC concentrations with increasing HCl concentration (Figure 5A). These changes are only limited by complete protonation of the surface amines.

The increase in $\mathrm{HCl}$ concentration also led to higher chiral nematic pitch values (Figure $5 \mathrm{C}$ ), since larger $D_{\text {eff }}$ results in the ChNC to be spaced further apart and thus weakening any chiral interactions. ${ }^{31}$ However, when considering these results, it is important to consider that the surface charge rises non-linearly with increasing $\mathrm{HCl}$ concentration resulting in the observed liquid crystalline behavior (Figure 5A, C) . 


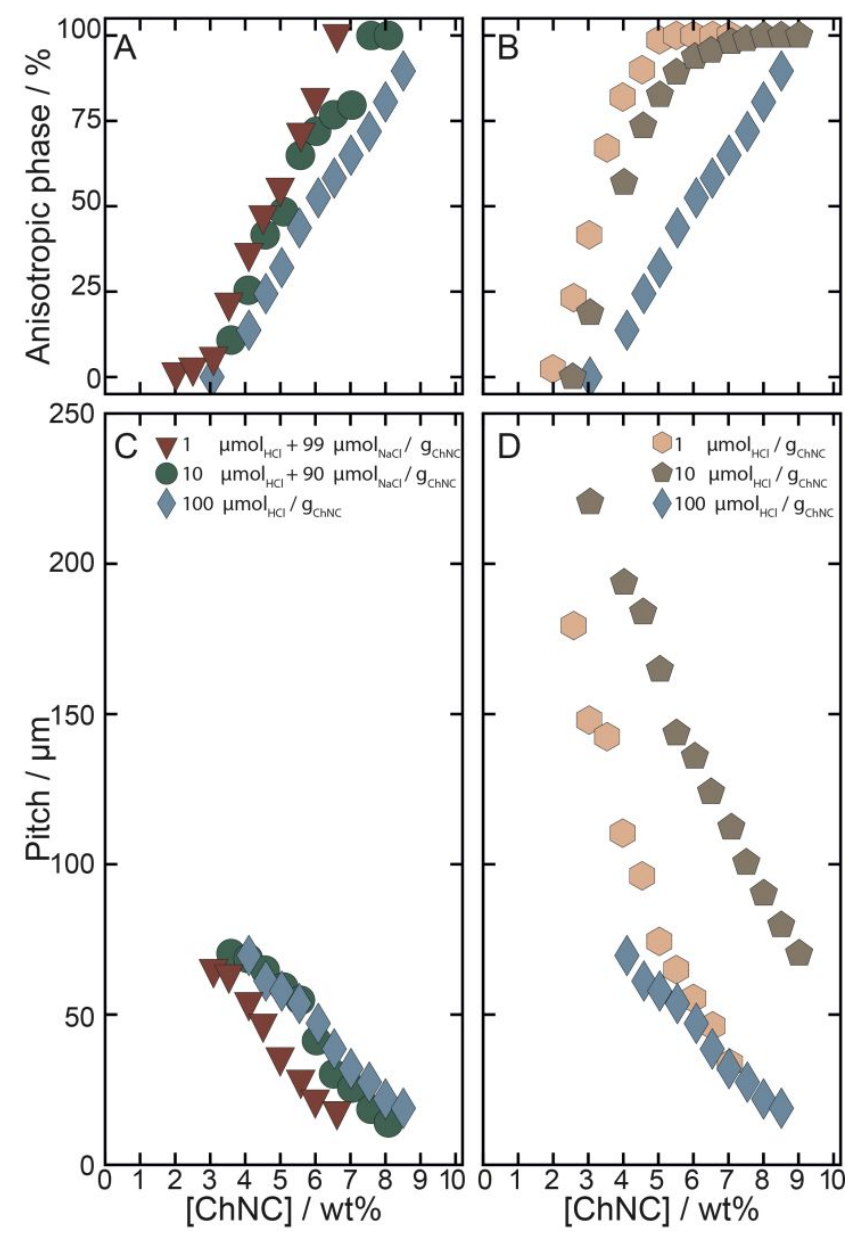

Figure 5. Effect of acidity and ionic strength on ChNC selfassembly. (A, B) Phase diagrams and (C, D) corresponding pitch diagrams showing the ChNC dependence with varying $\mathrm{HCl}$ concentration from $1-100 \mu m o l / g C h N C$, with the total ionic strength (A, C) standardized to $100 \mu$ mol/gChNC with NaCl or (B, D) uncorrected. 
The influence of $\mathrm{HCl}$ concentration was also tested without compensating for changes in the ionic strength. Therefore, the $D_{\text {eff would }}$ respond to the increase of both the surface charge and total ionic strength. In this case, three samples with HCl concentration of 1,10 , and $100 \mu m o l / g_{\mathrm{ChNC}}$ were prepared and so the total ionic strength is also different in each sample. The phase diagrams indicate that the first and second threshold concentrations increased with the total HCl concentration (Figure 5B). This can be explained by a higher ionic strength leading to a smaller Debye length and $D_{\text {eff }}$, in agreement with Eq. (1). The deviation from a linear trend when approaching the second threshold concentration is observed at the two lower $\mathrm{HCl}$ concentrations and it is possible that the chitin nanocrystal contribution becomes non-negligible at these relatively low ionic strength conditions.

The chiral nematic pitch reported in Figure 5D shows more dramatic variations. Increasing the ionic strength from 1 to 10 umol/g chNc resulted in a larger pitch but with a further increase from 10 to $100 \mathrm{mmol} / \mathrm{g}_{\mathrm{ChNC}}$ the pitch substantially dropped. The initial increase of the pitch corresponds to the increase of the surface charge of the ChNCs as more amine groups are protonated, while the ionic strength remains very low (at $10 \mu m o l / g_{\mathrm{ChNC}}$ and 10 wto ChNCs, $I=1 \mathrm{mM})$. Thus, the increase in the surface charge had a dominant effect, compared to the increase in the 
ionic strength. This led to ChNC being spaced apart more and thus weakening their chiral interactions, ultimately producing bigger chiral nematic pitch. However, when the amount of HCl was elevated from 10 to $100 \mu \mathrm{mol} / \mathrm{g}_{\mathrm{ChNC}}$, the surface charge of the ChNCs only slightly increased, thus the increase in the ionic strength became the dominant factor. Therefore, the Debye length strongly shrank (e.g., from $\sim 9.6 \mathrm{~nm}$ at $1 \mathrm{mM}$ to $\sim 3.0 \mathrm{~nm}$ at $10 \mathrm{mM})$. This caused a strong decrease in $D_{\text {eff }}$ so that the ChNCs could come in closer proximity and so strengthening their chiral interactions.

Solid films. The chiral nematic order can be preserved in the solid-state by evaporating the ChNC suspensions, giving rise to transparent, nanostructured films (Figure 1E). Cross-sectional SEM analysis revealed a well-ordered helicoidal structure with characteristic Bouligand arches (Figure 6A, 6B). ${ }^{32}$

The pitch of the helicoidal structures in dry films is determined by two processes: the self-assembly of ChNC in the chiral nematic phase, and the distortion experienced upon further drying after the onset of the kinetic arrest.2,33,34 The former depends primarily on the chNC concentration and the parameters explored above, whereas the factors affecting the onset of the kinetic arrest are less clear. Nevertheless, at a given ChNC concentration, the system will turn from liquid-like to solid-like, preventing ChNC rods from rearranging 
collectively. ${ }^{2}$ Past this point, further water evaporation leads to a vertical compression of the suspension ${ }^{35}$, forcing the ChNCs to get closer and reducing drastically the pitch. Since both the ionic strength and the $\mathrm{pH}$ influence the pitch before the kinetic arrest as well as the onset of the kinetic arrest itself, they represent the two main parameters controlling the final pitch of the film. Combining various $\mathrm{pH}$ and ionic strengths, it is possible to produce ChNC films with a helicoidal arrangement and pitch values ranging from $650 \mathrm{~nm}$ to $3720 \mathrm{~nm}$. Interestingly, ChNC suspensions could even be dried in a few hours at $90{ }^{\circ} \mathrm{C}$ and still yield films with a well ordered helicoidal structure (Figure S10).

The correspondence between the pitch in ChNC suspensions and in dry films is presented in a log-log plot (Figure 6C). ${ }^{33}$ The pitch in the liquid phase is measured from ChNC suspension in capillaries (cf. Figure 5) while the solid state (i.e. 100 wto ChNC concentration) is measured by cross-sectional SEM analysis of dry films. This plot shows that in general the ChNC suspensions that exhibit the largest pitch in solution correspond to the largest pitch in the solid state. However, to have full control over the pitch in the dry film, a better understanding of the onset of the kinetic arrest should be investigated in the future. 


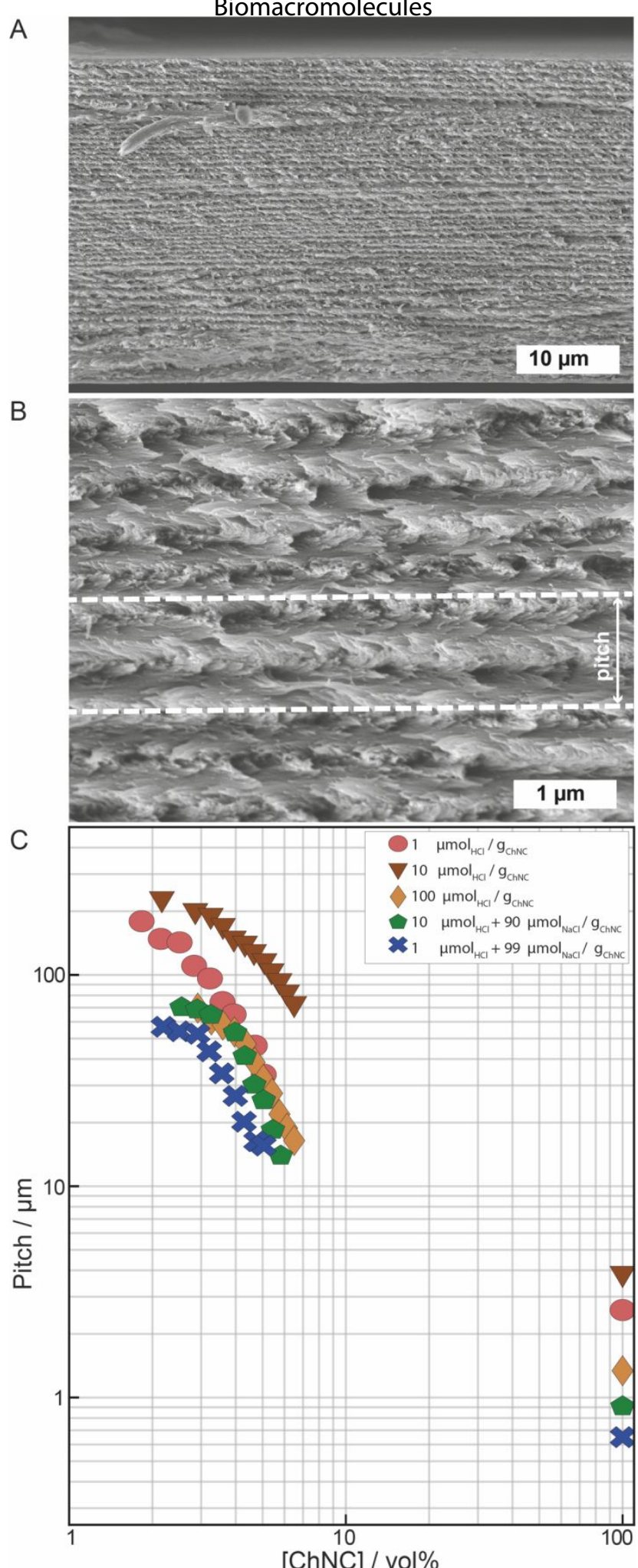

-

Figure 6. Cross-sectıonal St'M analysıs of chNc tilms at different conditions. Films prepared by drying chiral nematic ChNC suspension retain a helicoidal architecture (A), with Bouligand arches clearly seen at higher magnification (B). The chiral nematic pitch in suspension (as measured in capillaries) and in solid state (i.e. 100 wto ChNC concentration) can be tuned by varying the $\mathrm{HCl}$ and $\mathrm{NaCl}$ ACS Paragon Plus Environment concentration (C) . 


\section{CONCLUSIONS}

Here we demonstrated that a careful investigation of ChNC extraction methods, namely, chitin hydrolysis duration and acidity, tip sonication energy, degree of acetylation, ionic strength and $\mathrm{pH}$ led to a better understand of their colloidal properties and in turn allowed for control over their liquid crystalline behavior. Using this understanding, we were able to produce well-ordered helicoidal ChNC films with tunable pitch spanning an order of magnitude.

More specifically, we evaluated how the ChNC colloidal properties are affected by the preparation method and demonstrated that $3.0 \mathrm{M} \mathrm{HCl}$ at reflux for $270 \mathrm{~min}$ is sufficient to hydrolyze chitin to achieve a stable chNC suspension, with higher $\mathrm{HCl}$ concentrations only accelerating the reaction speed, while longer hydrolysis time, as in 3M540, yielded smaller, more charged and less polydisperse particles. Additionally, we addressed the effects of tip sonication energy, which assists in dispersing ChNC in a controlled manner and showed influence on the chiral nematic phase. The degree of acetylation prior to hydrolysis proved to be a way to reduce chNC width rather than increase the surface charge, while the ionic strength and $\mathrm{pH}$ allowed to finely tune ChNC self-assembly reaching chiral nematic pitch values as high as $250 \mu \mathrm{m}$. This understanding led to a controlled production of dried ChNC films that retained a 
helicoidal nanostructure. The pitch of dry helicoidal ChNC films can be tuned independently from the preparation conditions, via ionic strength or pH.

In conclusion, we believe that this comprehensive study with detailed information on the characterization and preparation of ChNC will serve well in promoting this exciting field and thus unlock the potential of chitin as a truly valuable colloidal system, due to its abundance and renewability as well as for its fascinating surface chemistry and liquid crystalline behavior.

\section{ASSOCIATED CONTENT}

\section{SUPPORTING INFORMATION}

The Supporting Information is available free of charge on the ACS Publications website.

An extensive description of all the experimental methods. Supplementary results: FT-IR, ssNMR, PXRD data showing chemical composition; conductometric titration, UV-Vis spectroscopy, extensive TEM data analysis supporting claims about ChNC colloidal properties; SEM images of ChNC suspensions dried at elevated temperature (Supporting_Information.pdf).

Additional data relating to this publication is available from the University of Cambridge data repository (https://doi.org/10.17863/CAM.40425). 


\title{
AUTHOR INFORMATION
}

\section{Corresponding Author}

*E-mail: sv319@cam.ac.uk

Current Addresses

tUniv. Grenoble Alpes, CNRS, CERMAV, Grenoble 38000, France

\begin{abstract}
Author Contributions
All authors have given approval to the final version of the manuscript.
\end{abstract}

\section{Funding Sources}

This work was supported by the European Research Council [ERC2014-STG H2020 639088], the BBSRC David Phillips Fellowship $[\mathrm{BB} / \mathrm{K} 014617 / 1]$, the EPSRC [EP/N509620/1], and Lord Lewis Research Studentship in Chemistry.

\author{
Notes \\ The authors declare no competing financial interest.
}

\section{ACKNOWLEDGMENT}

ACS Paragon Plus Environment 
A.N. and L.M.S. thank Dr David G. Reid, Ieva Goldberga, Rui Li and Dr Wai Ching Veronica Wong for valuable discussions and help with solid-state NMR, and Jordi Ferrer-Orri for providing an illustration of a shrimp.

REFERENCES

(1) Hamley, I. W. Liquid Crystal Phase Formation by Biopolymers. Soft Matter 2010, 6 (9), 1863-1871.

(2) Parker, R. M.; Guidetti, G.; Williams, C. A.; Zhao, T.; Narkevicius, A.; Vignolini, S.; Frka-Petesic, B. The SelfAssembly of Cellulose Nanocrystals: Hierarchical Design of Visual Appearance. Adv. Mater. 2018, 30 (19), 1704477.

(3) Revol, J. F.; Marchessault, R. H. In Vitro Chiral Nematic Ordering of Chitin Crystallites. Int. J. Biol. Macromol. 1993, $15(6), 329-335$.

(4) Habibi, Y.; Lucia, L. A.; Rojas, O. J. Cellulose Nanocrystals: Chemistry, Self-Assembly, and Applications. Chem. Rev. 2010, 110 (6), 3479-3500.

(5) Dong, S.; Roman, M. Fluorescently Labeled Cellulose Nanocrystals for Bioimaging Applications. J. Am. Chem. Soc. 2007, $129(45), 13810-13811$.

(6) Kaushik, M.; Basu, K.; Benoit, C.; Cirtiu, C. M.; Vali, H.; Moores, A. Cellulose Nanocrystals as Chiral Inducers: Enantioselective Catalysis and Transmission Electron 
Microscopy 3D Characterization. J. Am. Chem. Soc. 2015, 137 (19), 6124-6127.

(7) Li, J.; Revol, J.-F.; Marchessault, R. H. Effect of Degree of Deacetylation of Chitin on the Properties of Chitin Crystallites. J. Appl. Polym. SCi. 2004, 65 (2), 373-380.

(8) Belamie, E.; Davidson, P.; Giraud-Guille, M. M. Structure and Chirality of the Nematic Phase in $\alpha$-Chitin Suspensions. J. Phys. Chem. B 2004, 108 (39), 14991-15000.

(9) Rinaudo, M. Chitin and Chitosan: Properties and Applications. Prog. Polym. Sci. 2006, 31 (7), 603-632. Zavattieri, P. Twisting Cracks in Bouligand Structures. J. Mech. Behav. Biomed. Mater. 2017, 76 (March), 38-57.

Liquid Crystal Systems from Fibrillar Polysaccharides. Nature 1959, $184(4686), 632-633$. Properties of Aqueous Suspensions of Chitin Crystallites. J. Colloid Interface Sci. 1996, 183 (2), 365-373. Colloidal Particles. Ann. N. Y. Acad. Sci. 1949, 51 (4), $627-659$ Dong, X. M.; Kimura, T.; Revol, J.-F.; Gray, D. G. Effects of Ionic Strength on the Isotropic-Chiral Nematic 
Phase Transition of Suspensions of Cellulose Crystallites. Langmuir 2002, 12 (8), 2076-2082.

Stroobants, A.; Lekkerkerker, H. N. W.; Odijk, T. Effect of Electrostatic Interaction on the Liquid Crystal Phase Transition in Solutions of Rodlike Polyelectrolytes. Macromolecules 1986, 19 (8), 2232-2238.

Sato, T.; Teramoto, A. Perturbation Theory of Isotropic-Liquid-Crystal Phase Equilibria in Polyelectrolyte Solutions. Phys. A Stat. Mech. its Appl. 1991, 176 (1), 7286.

Belamie, E.; Mosser, G.; Gobeaux, F.; Giraud-Guille, M. M. Possible Transient Liquid Crystal Phase during the Laying out of Connective Tissues: $\alpha$-Chitin and Collagen as Models. J. Phys. Condens. Matter 2006, 18 (13), S115. Tzoumaki, M. V.; Moschakis, T.; Biliaderis, C. G. Metastability of Nematic Gels Made of Aqueous Chitin Nanocrystal Dispersions. Biomacromolecules 2010, 11 (1), $175-181$

Nanocrystals Prepared from Shrimp Shells and Their Specific Properties. Biomacromolecules 2007, 8 (8), 252- 257.

(20) O'Connor, C. Acidic and Basic Amide Hydrolysis. $Q$. Rev. Chem. Soc. 1970, 24 (4), 553-564.

(21) Krieble, V. K.; Holst, K. A. Amide Hydrolysis with 
High Concentrations of Mineral Acids. J. Am. Chem. Soc. $1938,60(12), 2976-2980$. Lee, S. D. A Numerical Investigation of Nematic Ordering Based on a Simple Hard-Rod Model. J. Chem. Phys. $1987,87(8), 4972-4974$.

J.; Park, J. H.; Scalia, G.; Bergström, L. Cellulose Nanocrystal-Based Materials: From Liquid Crystal SelfAssembly and Glass Formation to Multifunctional Thin Films. NPG Asia Mater. 2014, 6 (1), 1-12.

Separation Behavior in Aqueous Suspensions of Bacterial Cellulose Nanocrystals Prepared by Sulfuric Acid Treatment. Langmuir 2008, 25 (1), 497-502.

(25)

R.; Osipov, M. A.; Baller, J.; Lagerwall, J. P. F. Fractionation of Cellulose Nanocrystals: Enhancing Liquid Crystal Ordering without Promoting Gelation. NPG Asia Mater. 2018, $10(5), 455-465$. Reflection Wavelength of Iridescent Solid Films of Nanocrystalline Cellulose. Biomacromolecules 2011, 12 (1), $167-172$

Nguyen, T. D.; Maclachlan, M. J. Biomimetic Chiral 
Nematic Mesoporous Materials from Crab Cuticles. Adv. Opt. Mater. 2014, 2 (11), 1031-1037.

(28) Elazzouzi-Hafraoui, S.; Nishiyama, Y.; Putaux, J.-L.; Heux, L.; Dubreuil, F.; Rochas, C. The Shape and Size Distribution of Crystalline Nanoparticles Prepared by Acid Hydrolysis of Native Cellulose. Biomacromolecules 2008, 9 (1), $57-65$.

Structure and Chirality of the Nematic Phase in $\alpha$-Chitin Suspensions. J. Phys. Chem. B 2004, 108 (39), 14991-15000.

Effect of Electrostatic Interaction on Phase separation Behaviour of Chitin Crystallite Suspensions. Int. J. Biol. Macromol. 1996, 18 (3), 177-187.

a Cellulose Microcrystal Suspension by Poly (Ethylene Glycol) Grafting. Langmuir 2001, 17 (1), 21-27. Bouligand, y. Sur Une Architecture Torsadée Répandue Dans de Nombreuses Cuticules d'Arthopodes. C. R. Hebd. Seances Acad. Sci. 1965, 261, 3665-3666. Parker, R. M.; Frka-Petesic, B.; Guidetti, G.; Kamita, G.; Consani, G.; Abell, C.; Vignolini, S. Hierarchical SelfAssembly of Cellulose Nanocrystals in a Confined Geometry. ACS Nano 2016, 10 (9), 8443-8449. 
(34) Frka-Petesic, B.; Guidetti, G.; Kamita, G.; Vignolini, S. Controlling the Photonic Properties of Cholesteric Cellulose Nanocrystal Films with Magnets. Adv. Mater. 2017, $29(32), 1-7$.

(35) Frka-Petesic, B.; Kamita, G.; Guidetti, G.; Vignolini,

S. Angular Optical Response of Cellulose Nanocrystal Films Explained by the Distortion of the Arrested Suspension upon Drying. Phys. Rev. Mater. 2019, 3 (4), 045601.

\section{Table of Content graphic}

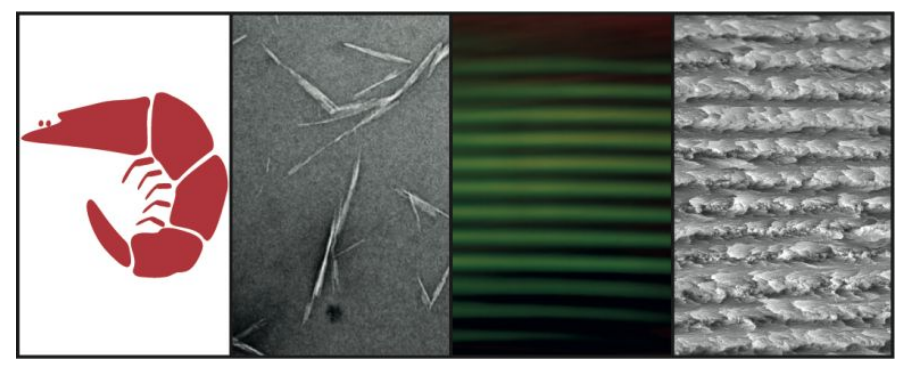

